\title{
Hybrid Neurosurgeon: Panacea to Debate on Coiling versus Clipping
}

\author{
Abrar Ahad Wani ${ }^{1}$ Mohsin Bhat ${ }^{1}$ \\ ${ }^{1}$ Department of Neurosurgery, Sherikashmir Institute of Medical \\ Sciences (SKIMS), Srinagar, Jammu and Kashmir, India
}

Indian J Neurosurg 2018;7:79-80

The advent of the endovascular surgery dates back to 1995 when the Guglielmi Detachable coils were approved by the Food and Drug Administration (FDA). ${ }^{1}$ This fascinating new approach to the neurovascular disease facilitated the treatment of the same vascular lesion via an endoluminal technique within the blood vessel. The realization of less morbidity and mortality by this approach due to its minimal invasive nature has made this technique a favorable one in treating most of the neurovascular diseases. Simultaneous trials began to validate the efficacy and safety of this new modality in aneurysm treatment, and in a short span of 10 years, the new modality shined enough to start eclipsing the gold standard procedure of the time, namely "clipping." ISAT (International Subarachnoid Aneurysm Trial) and BRAT (Barrow Ruptured Aneurysm Trial) trials started the discussion of coiling versus clipping, and the evidence-based studies started leaning in favor of coiling. The response of the surgical community was natural, and reactionary opposition as the coiling was not done by most neurosurgeons. ${ }^{2-5}$ The situation seemed ripe for the pharmaceutical companies that started investing huge amounts of money to develop the hardware needed, and this led to the rapid advancement in the endovascular techniques. Technological advances included a variety of the devices incorporated in the armamentarium, including various types of coils, balloon catheters, access catheters, embolization materials, flow diverters, stents, and so on. On the other hand, surgical techniques of aneurysm coiling remained static and nothing significant changed over the last couple of decades. The spectrum of the vascular lesions treated by the endovascular intervention progressively increased from coiling of aneurysms to management of fistula, arteriovenous malformations, vascular tumors, and stroke. However, the application of this technique to the aneurysm revolutionized the approach by which an aneurysm is dealt in an altogether different way. Analyzing the inevitable takeover by endovascular modalities, many neurosurgeons started training in this new specialty with the concept that they will be better than others in managing aneurysm. This assumption was based on the fact that all the patients of subarachnoid hemorrhage (SAH) have been managed by neurosurgeons for decades, be it simple clipping or any complication of the SAH-like hydrocephalus, hematoma evacuation, or decompressive craniotomy-as the need may be. Even in many salvage procedures following complications in coiling, a neurosurgeon has to step in. Therefore, endovascular technique started becoming a popular choice among neurosurgical residents. Incorporation of the basic endovascular skills in the curriculum of the trainees is the need of the hour. The American Board of the Neurosurgical Survey has already made this mandatory. Residents should be well acquainted with the knowledge of the neurovascular anatomy and be familiar with the basic devices and angiographic equipment. They should be able to perform four-vessel angiogram and be research oriented. These basic skills will help the trainees master the technique quickly in their future fellowship. Conversely, the residents who will not acquire basic endovascular skills have to overcome the steep learning curve and may not be able to perform the endovascular procedures by the completion of their fellowship program. ${ }^{6}$

The need of the hour is the making of the hybrid neurosurgeon who is well versed in both microvascular surgery and endovascular techniques. The important point to mention is that the two techniques should not be viewed as diametrically opposite treatment modalities but collaborative and supplemented taking into full consideration the natural history of the disease and the risk-benefit ratio of the procedure. To achieve competence, trainees will require an exposure to a variety of the vascular lesions during training along with adjuncts like training in cadaveric dissection, microsurgical and endovascular skill laboratories, and animal procedure simulators. This issue needs to be addressed in the new training program. Most neurosurgeons perceive and consider microsurgical procedure to have a steeper learning curve than endovascular techniques; thus, the hybrid neurosurgeon shows a greater tendency toward endovascular technique due to the less time required to master. Equal time and training skills should be provided to trainees in both techniques to eliminate this bias.

\section{Address for correspondence}

Abrar Ahad Wani, MS, MCh, Department of Neurosurgery, Sherikashmir Institute of Medical Sciences (SKIMS), Srinagar, Jammu and Kashmir, India

(e-mail: drabrarahadwani@gmail.com).
DOI https://doi.org/

10.1055/s-0038-1667282.

ISSN 2277-954X.
C2018 Neurological Surgeons'

Society of India
License terms

$($ () (1) $\Theta \circledast$ 
Chalouhi et al studied the preparedness of U.S. neurosurgery graduate trainees for neuroendovascular fellowship. This study's results suggest potential gaps in the training of neurosurgery residents regarding endovascular neurosurgery. In an era of minimally invasive therapies, changes in residency curricula may be needed to keep pace with the ever-changing field of neurosurgery. ${ }^{7}$ In our institute, once our department decided to start the program, I and one more faculty member went for training in the endovascular procedures. Since then we could start coiling of the aneurysms and our residents are now getting the training simultaneously in both conventional neurosurgery and endovascular surgery.

Most studies and the research in Europe and North America regarding the incorporation of dual-training program in the curriculum of the neurosurgical residents have proven to be beneficial. The landscape of the neurovascular disease is ever changing. Therefore, there has to be a rapid paradigm shift in the training and the resident education. Although the hybrid training is a norm in North America, in our country, the integration of such endovascular surgery is still in infancy. The complexity of the microsurgical technique with time restriction has made it intensely difficult to concentrate on both fields. It is an uphill task to master both techniques during training so what seems feasible is learning the basic skill of both during the training program. This can be accomplished by making it mandatory for neurosurgical trainees to learn four-vessel angiogram, be well versed in the neurosurgical anatomy, and acquire learning skill in the endovascular and microsurgical laboratory. However, this requires a good financial support and sponsorship, which can be a major obstacle in a developing country such as India. These training programs should be scrutinized and monitored and critical evaluation of the outcome should be done. ${ }^{6,7}$

\section{Conclusion}

In developing countries, in both the fields of preparing the curriculum and monitoring the training program for hybrid neurosurgeons, various neurosurgical societies in the country can take a lead role. This is important, as the clipping will continue to be used in most patients, as the affordability is a major concern in this part of the world. Nevertheless, the growing middle class will be the patient population who will be moving toward endovascular interventions as the demand for minimally invasive procedures is growing in all the surgical specialties and neurosurgery is no exception. The ultimate goal should be to make a proficient hybrid neurosurgeon, one who would be well versed in all the treatment modalities and their consequences. This can be achieved by extensive endovascular and microsurgical exposure during training. Ultimately, the progression to mastery of the expertise will depend on the dedication of the concerned surgeon.

\section{References}

1 Morrison SR. Guglielmi detachable coils: an alternative therapy for surgically high-risk aneurysms. J Neurosci Nurs 1997;29(4):232-237

2 Molyneux AJ, Kerr RS, Yu LM, et al; International Subarachnoid Aneurysm Trial (ISAT) Collaborative Group. International subarachnoid aneurysm trial (ISAT) of neurosurgical clipping versus endovascular coiling in 2143 patients with ruptured intracranial aneurysms: a randomised comparison of effects on survival, dependency, seizures, rebleeding, subgroups, and aneurysm occlusion. Lancet 2005;366(9488):809-817

3 Mitchell P, Kerr R, Mendelow AD, Molyneux A. Could late rebleeding overturn the superiority of cranial aneurysm coil embolization over clip ligation seen in the International Subarachnoid Aneurysm Trial? J Neurosurg 2008;108(3):437-442

4 Spetzler RF, McDougall CG, Zabramski JM, et al. The Barrow Ruptured Aneurysm Trial: 6-year results. J Neurosurg 2015;123(3):609-617

5 Ausman JI. The International Subarachnoid Aneurysm Trial II: comparison of clipping vs coiling: key questions. Are the results of the study generalizable? Should clipping be done for patients less than 40 years of age? Surg Neurol 2008;70(1):104-107

6 Chowdhry SA, Spetzler RF. Genealogy of training in vascular neurosurgery. Neurosurgery 2014;74(1, Suppl 1):S198-S203

7 Chalouhi N, Zanaty M, Tjoumakaris S, et al. Preparedness of neurosurgery graduates for neuroendovascular fellowship: a national survey of fellowship programs. J Neurosurg 2015;123(5):1113-1119 